\title{
Apparent depth from progressive exposure of moving shadows: The kinetic depth effect in a narrow aperture
}

\author{
R. H. DAY \\ Monash University, Clayton, Victoria, Australia
}

\begin{abstract}
The kinetic depth effect refers to the three-dimensional appearance of a two-dimensional moving shadow cast by a three-dimensional object rotating in depth on a translucent screen. Informal observations indicated that the appearance of depth is strong and convincing when the moving shadow is progressively exposed in a narrow aperture in the manner of the Zöllner-Parks effect. These observations were confirmed in two experiments with two- and three-dimensional "skeletal" objects. Apparent depth of the objects and of their movement was reported both when the fractionally visible shadows passing across the aperture were symmetrical and when they were asymmetrical about their vertical axes. These data show that information about the depth of an object and its movement is integrated over time, presumably in a similar manner to outline shape in the earlier Zöllner-Parks studies.
\end{abstract}

If objects rotating in depth relative to an observer are presented in parallel projection on one side of a translucent screen, the resultant two-dimensional (2-D) shadows on the other side of the screen are readily seen as the original objects rotating in depth. This effect was first reported by Miles (1931) for the projection of a rotating two-bladed fan, and shortly thereafter by Metzger (1934) for that of vertical rods mounted on a rotating turntable. The effect was later studied in detail by Wallach and O'Connell (1953) with a variety of skeletal and solid objects. They called the appearance of depth in the 2-D projected shadows the kinetic depth effect (KDE) and concluded that it is determined mainly by simultaneous changes in the length and orientation of projected contours.

A good deal of recent research on the KDE has focused on the minimal conditions for the recovery of depth from 2-D displays. For example, Braunstein, Hoffman, Shapiro, Anderson, and Bennett (1987) used 3-D displays that were reduced to a few bright points and were observed in a limited range of views. It is shown here that the KDE occurs strongly and consistently when only a narrow vertical section of the moving projection is visible at any instant as it passes behind a slit-like aperture in the manner of the Zöllner-Parks effect (Parks, 1965; Zöllner, 1862). Like the Zöllner-Parks effect with a 2-D planar figure, the parallel projection of a 3-D object is seen as complete.

The idea for progressively exposing the projection of a rotating object in a narrow aperture arose in the course of discussions of the KDE and the Zöllner-Parks effect with Emily Bushnell and B. E. McKenzie during one of a series of seminars on perception in early infancy in 1988. The contributions of both colleagues are gratefully acknowledged. The assistance of Richard Hobbs, Vladimir Kohout, and Rosemary Williams in technical matters, and of Fiona Duffy in the collection and analysis of data is gratefully acknowledged. Address correspondence to $\mathbf{R}$. $\mathbf{H}$. Day, Department of Psychology, Monash University, Clayton, Victoria 3168, Australia.
Of particular interest, however, is that the 3-D character both of the original object and of its motion is readily recovered from the progressive exposure of the 2-D image moving in the elongated aperture. As far as is known, apparent depth under such reduced conditions has not been described before.

In the present paper, two experiments are reported. They involved binocular observation of parallel projections in a narrow vertical aperture of 2-D and 3-D "skeletal" objects when they were stationary and when they were rotating about their vertical axes. The subjects sat with their eyes $200 \mathrm{~cm}$ from a mid-gray opaque screen $100 \mathrm{~cm}$ wide and $43 \mathrm{~cm}$ high mounted on a table (see Day $\&$ Duffy, 1988). A central aperture $2 \mathrm{~cm}$ wide extended from the top to the bottom of the screen. The subject's eyes were on a level with the center of the aperture. Immediately behind the aperture was a sheet of translucent drawing paper stretched across a square frame. The stems of the stimulus objects (Figure 1) were fixed firmly in a cylindrical holder which was either stationary or rotated by means of a silent motor at $9.5 \mathrm{rpm}$. Clear, sharp shadows of the stimulus objects were cast on the translucent screen by means of a projector $350 \mathrm{~cm}$ from the vertical stems to which the objects were attached. Thus, at any moment the subjects saw a narrow $(2-\mathrm{cm})$ vertical section of the planar shadow which was either stationary or moving across the aperture as the object rotated.

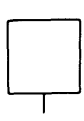

A

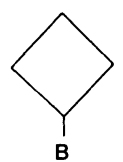

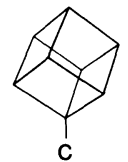

C

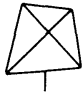

D

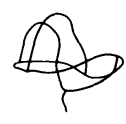

E
Figure 1. The five objects of which parallel projections (shadows) were viewed in the two experiments. 
Preliminary observations showed that the KDE occurred when the shadows moved across the aperture. The purpose of the first experiment was formally to check the occurrence of the KDE with the parallel projections of 2-D and 3-D objects. The five objects, two 2-D regular objects (square and diamond), two 3-D regular objects (cube and "wedge"), and a 3-D irregular object, are shown in that order in Figures 1A-1E. They were constructed of 2-mm welding rod. The square sections in Figures $1 \mathrm{~A}, 1 \mathrm{~B}$, and $1 \mathrm{C}$ were $12 \mathrm{~cm}$ along a side, and the sides of the triangular sections in Figure 1D were $14 \mathrm{~cm}$ long. The 3-D irregular object (Figure 1E) was made by bending lengths of welding rod and had a maximum vertical extent of $12 \mathrm{~cm}$ and a maximum horizontal extent of $20 \mathrm{~cm}$. The objects were positioned so that 9$\mathrm{cm}$ shadows of their vertical stems were centered in the lower part of the aperture.

The five stationary shadows followed by the five moving shadows were presented in random order to 10 subjects (graduate students, teaching assistants, and senior undergraduates), none of whom had observed the KDE in an experiment before. They were asked simply to describe the shadow, first when it was stationary and then when it was moving as the object rotated. If, under each condition, it was unclear whether the shadow itself or its movement was perceived as 2-D or 3-D, the subject was questioned directly on each point at the end of the session.

The results are shown for the stationary and moving shadows in Table 1. Whereas the shadows for the 2-D objects were seen as 2-D under both conditions by all sub-

Table 1

Number of Subjects Reporting the Stationary and Moving Shadows in the Aperture as Two-Dimensional (2-D) or Three-Dimensional (3-D) Objects and Their Movement as Two-Dimensional or Three-Dimensional in the Two Experiments

\begin{tabular}{|c|c|c|c|c|}
\hline \multirow[b]{2}{*}{ Object } & \multirow{2}{*}{$\begin{array}{l}\text { Apparent } \\
\text { Depth }\end{array}$} & \multicolumn{2}{|c|}{ Figure } & \multirow[b]{2}{*}{ Movement } \\
\hline & & Stationary & Moving & \\
\hline
\end{tabular}
Experiment 1

$\begin{array}{lrrrr}\text { 2-D Square } & \text { 2-D } & 10 & 10 & 0 \\ & \text { 3-D } & 0 & 0 & 10 \\ \text { 2-D Diamond } & \text { 2-D } & 10 & 10 & 0 \\ & \text { 3-D } & 0 & 0 & 10 \\ \text { 3-D Cube } & \text { 2-D } & 7 & 1 & 0 \\ & \text { 3-D } & 3 & 9 & 10 \\ \text { 3-D Wedge } & \text { 2-D } & 10 & 1 & 0 \\ & \text { 3-D } & 0 & 9 & 10 \\ \text { 3-D Irregular } & \text { 2-D } & 7 & 1 & 0 \\ & \text { 3-D } & 3 & 9 & 10\end{array}$

Experiment 2

\begin{tabular}{lrrrc} 
2-D Square & 2-D & 10 & 10 & 0 \\
& 3-D & 0 & 0 & 10 \\
2-D Diamond & 2-D & 10 & 8 & 0 \\
& 3-D & 0 & 2 & 10 \\
3-D Cube & 2-D & 8 & 3 & 0 \\
& 3-D & 2 & 7 & 10 \\
3-D Wedge & 2-D & 8 & 3 & 0 \\
& 3-D & 2 & 7 & 10 \\
3-D Irregular & 2-D & 4 & 0 & 0 \\
& 3-D & 6 & 10 & 10 \\
\hline
\end{tabular}

jects, those for the 3-D objects were seen by either 7 or 10 subjects as 2-D when stationary, but as 3-D by 9 of the 10 subjects when moving. The movement of all shadows, regardless of whether they were cast by 2-D or 3-D objects, was seen as 3-D. It can be noted that movement was seen as rotation in one or the other direction in depth or alternatingly in both directions, that is, as oscillation of direction in depth.

These data confirm the preliminary observations and convincingly show that the KDE occurred for almost all subjects when, from moment to moment, only the stems and a narrow vertical section of the parallel projections of rotating 2-D or 3-D objects were visible.

In the first experiment, the shadows of the vertical stems - the axes of rotation of the objects-were centered and were visible in the lower part of the aperture. The question therefore arose as to whether the KDE occurs when the stem is moved to one side of the aperture so that it is out of sight and the object is asymmetrically positioned relative to the aperture, rather than symmetrically as before. A second experiment was performed to answer this question. The screen was moved laterally so that the aperture was positioned halfway between the stem and the outer extremities of the object. For half of the 10 new subjects the aperture was moved to the right, and for half to the left. With this arrangement, both the stationary and moving shadows were different from those in the first experiment.

The results for Experiment 2 are also shown in Table 1. Although reports of apparent depth in the moving shadows were slightly less frequent than in the first experiment for Figures $1 \mathrm{~B}, 1 \mathrm{C}$, and $1 \mathrm{D}$, they were minimally greater for Figure 1E. As before, all 10 subjects reported the movement of the objects as 3-D.

The data from these two sets of observations are consistent in showing that the KDE occurs when moving planar shadows of rotating objects are only fractionally visible in an aperture and the complete shadow is exposed only progressively.

Three points can be made in conclusion. First, Parks (1965) pointed out that for a 2-D figure to be recognized and seen as complete when it is viewed passing behind a narrow aperture, the sequential views must somehow be stored, encoded, and reassembled in the original order. The same point can be made about the planar shadows of rotating objects. The significance of the present data is in showing that cues for depth in planar shadows, like cues for form, must be similarly processed to produce a recognizable 3-D object rotating in depth.

The second point concerns the storage and encoding of cues for depth other than those present in the shadows of rotating objects. It is reasonable to suppose that other cues (e.g., linear perspective, static overlay, and elevation in the visual field) might be processed in much the same way in aperture presentation. This point is of interest since virtually all experiments on the Zöllner-Parks effect to date have involved 2-D stimulus patterns. It seems likely, on the basis of the results reported here, 
that apparent depth would be evident if a picture or scene with cues for depth were to be passed across an aperture.

Finally, the effect described here suggests an alternative means of studying the minimal conditions for the occurrence of the KDE. By progressively narrowing the aperture and introducing periods of occlusion of the moving shadow during each complete revolution of the object, the amount of information for depth available at any moment could systematically be varied.

\section{REFERENCES}

Braunstein, M. L., Hoffman, D. D., Shapiro, L. R., Anderson, G. J., \& BENNETT, B. M. (1987). Minimum points and views for the recovery of three-dimensional structure. Journal of Experimental Psychology: Human Perception \& Performance, 13, 335-343.

DAY, R. H., \& DUFFY, F. M. (1988). Illusions of time and extent when the Müller-Lyer figure moves in an aperture. Perception \& Psychophysics, 44, 205-210.

METZGER, W. (1934). Tiefenericheinungen in optichen bewegungsfelden. Psychologische Forschung, 20, 195-260.

MiLES, W. R. (1931). Movement interpretation of the silhouette of a revolving fan. American Journal of Psychology, 43, 392-405.

PARKs, T. E. (1965). Post-retinal visual storage. American Journal of Psychology, 78, 145-147.

Wallach, H., \& O'Connell, D. N. (1953). The kinetic depth effect. Journal of Experimental Psychology, 45, 205-217.

ZöLLNER, F. (1862). Über eine neue Art anorthoskopischer Zerrbilder. Annallende Physik, Poggendorff's Annalen, 117, 477-484.

(Manuscript received December 28, 1988.) 